\title{
Exotic populations in globular clusters: blue stragglers as tracers of the internal dynamical evolution of stellar systems
}

\section{Francesco R. Ferraro}

\author{
Department of Physics and Astronomy, University of Bologna, Viale Berti Pichat 6/2, 40127- \\ Bologna, Italy \\ email: francesco.ferraro3@unibo.it
}

\begin{abstract}
In this paper I present an overview of the main observational properties of a special class of exotic objects (the so-called Blue Straggler Stars, BSSs) in Galactic Globular Clusters (GCs). The BSS specific frequency and their radial distribution are discussed in the framework of using this stellar population as probe of GC internal dynamics. In particular, the shape of the BSS radial distribution has been found to be a powerful tracer of the dynamical evolution of stellar systems, thus allowing the definition of an empirical "clock" able to measure the dynamical age of stellar aggregates from pure observational properties.
\end{abstract}

Keywords. globular clusters, blue stragglers, dynamics

\section{Introduction}

Globular clusters (GCs) are the only cosmic structures that within a time-scale shorter than the age of the Universe undergo nearly all the physical processes known in stellar dynamics (Meylan \& Heggie 1997). Gravitational interactions and collisions among single stars and/or binaries are expected to be quite frequent, especially in high density environments (e.g. Hut et al. 1992) characterizing the inner regions of GCs. Such a pronounced dynamical activity can generate populations of exotic objects, like X-ray binaries, millisecond pulsars and blue straggler stars (BSSs; see, e.g., Paresce et al. 1992, Bailyn 1995, Bellazzini et al. 1995, Ferraro et al. 2001, Ransom et al. 2005, Pooley \& Hut 2006, Ferraro et al. 2009a, Lanzoni et al. 2010).

Blue straggler stars (BSSs) are commonly defined as stars brighter and bluer than the main-sequence (MS) turnoff in the host stellar cluster. They are thought to be central Hburning stars, more massive than the MS turnoff stars (Shara et al. 1997, Gilliland et al. 1998, De Marco et al. 2004, Fiorentino et al. 2014). In stellar systems with no evidence of recent star formation, their origin cannot be explained in the framework of normal singlestar evolution. Two main formation channels are currently favored: (1) mass transfer (MT) in binary systems (McCrea 1964) possibly up to the complete coalescence of the two stars, and (2) stellar collisions (Hills \& Day 1976). Both these processes can potentially bring new hydrogen into the core and therefore "rejuvenate" a star to its MS stage (e.g., Lombardi et al. 1995, Lombardi et al. 2002; Chen \& Han 2009).

MT in binaries might be the dominant formation channels in all environments (e.g., Knigge et al. 2009; Leigh et al. 2013), and most likely it is so in low-density GCs, open clusters and the Galactic field (Ferraro et al. 2006a; Sollima et al. 2008; Mathieu et al. 2009; Gosnell et al. 2014; Preston \& Sneden 2000). Collisions are believed to be important especially in dense environments, such as the cores of globular clusters (GCs; Bailyn 1992; Ferraro et al. 1993, Ferraro et al. 1997, Ferraro et al. 2003a) and even the centre of some open clusters (Leonard \& Linnell 1992; Glebbeek et al. 2008). 


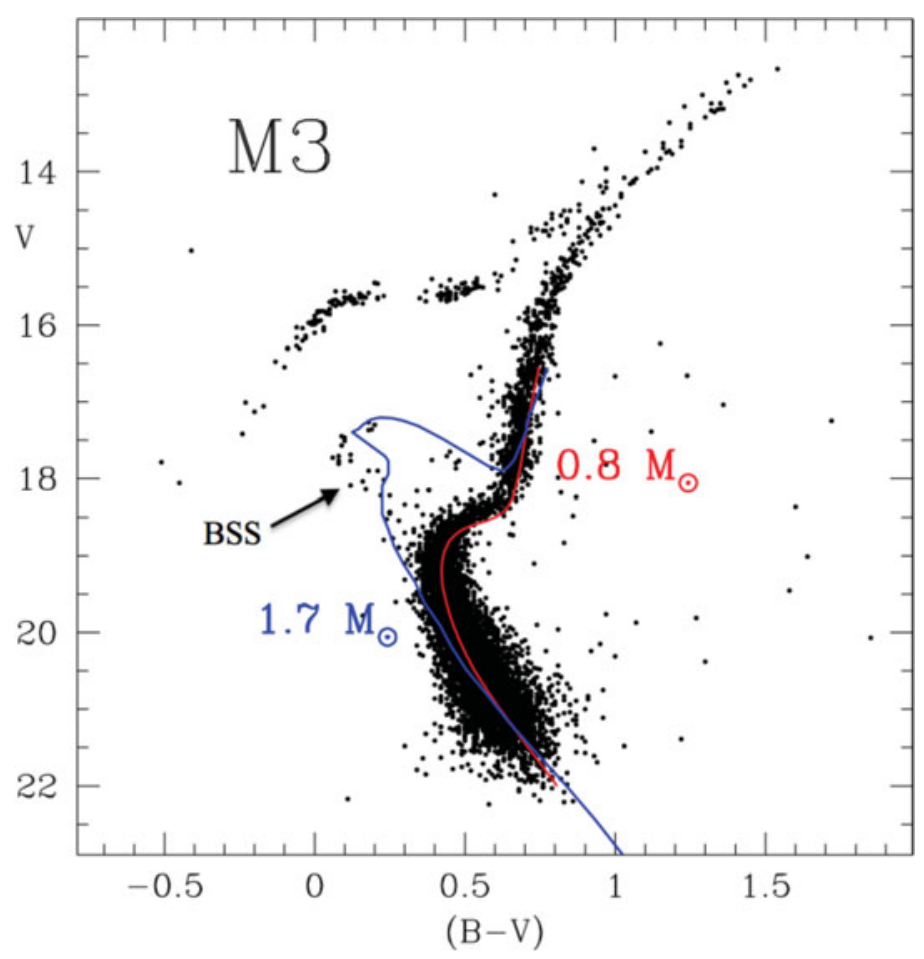

Figure 1. Optical CMD of the globular cluster M3, with the location of BSSs indicated by the arrow. The theoretical track corresponding to $0.8 M_{\odot}$ well reproduces the main evolutionary sequences of the cluster, while BSSs populate a region of the CMD where core hydrogen-burning stars of $\sim 1.7 M_{\odot}$ are expected. From Buonanno et al. (1994)

Because of their large number of member stars, GCs are the ideal environment for BSS studies. Nominally all the GCs observed so far have been found to harbor a significant number of BSSs (Piotto et al. 2004; Leigh et al. 2007). Moreover, the stellar density in GCs varies dramatically from the central regions to the outskirts, and since BSSs in different environments (low- versus high-density) could have different origins (e.g., Fusi Pecci et al. 1992; Ferraro et al. 1995, Davies et al. 2004), these stellar systems allow to investigate both formation channels simultaneously. However a clear distinction is hampered by the internal dynamical evolution of the parent cluster (Ferraro et al. 2012). In fact, having masses larger than normal cluster stars, BSSs are affected by dynamical friction, a process that drives the objects more massive than the average toward the cluster centre, over a timescale which primarily depends on the local mass density (e.g., Alessandrini et al. 2014). Hence, as the time goes on, heavy objects (like BSSs) orbiting at larger and larger distances from the cluster centre are expected to drift toward the core: as a consequence, the radial distribution of BSSs develops a central peak and a dip, and the region devoid of these stars progressively propagates outward.

Ferraro et al. (2012) used this argument to define the so-called "dynamical clock", an empirical tool able to measure the dynamical age of a stellar system from the shape of its BSS radial distribution. This appears indeed to provide a coherent interpretation of the variety of BSS radial distributions observed so far: GCs with a flat BSS radial distribution (Ferraro et al. 2006b; Dalessandro et al. 2008a; Beccari et al. 2011) are dynamically young systems, GCs with bimodal distributions (e.g., Ferraro et al. 1993, Ferraro et al. 2004; Lanzoni et al. 2007a; Dalessandro et al. 2008b; Beccari et al. 2013, and 


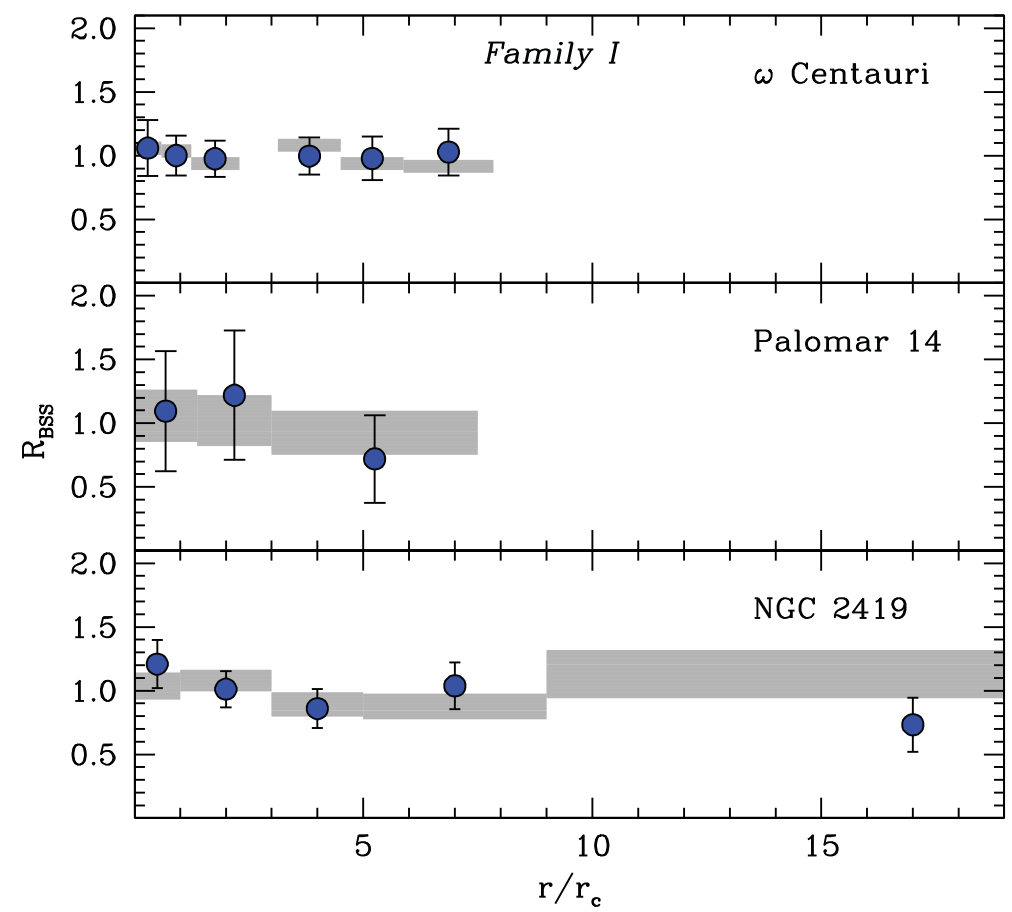

Figure 2. BSS radial distribution observed in $\omega$ Centauri, Palomar 14 and NGC 2419, with the large filled circles marking the values of the double normalized specific frequency $R_{\mathrm{BSS}}$. The distribution of the double normalized ratio measured for RGB or HB stars is also shown for comparison (grey strips). The BSS radial distribution is flat and totally consistent with that of the reference population, thus indicating a low degree of dynamical evolution for these three GCs (Family I). From Ferraro et al. (2012).

references therein) are dynamically intermediate-age systems (their actual dynamical age being determined by the distance of the dip of the distribution from the cluster centre), and GCs with a single-peaked BSS distribution (Ferraro et al. 1999a; Lanzoni et al. 2007b; Contreras Ramos et al. 2012; Dalessandro et al. 2013) are dynamically old systems.

\section{Setting the dynamical clock for stellar systems}

In order to perform meaningful comparisons among different clusters the first step is the definition of an appropriate BSS specific frequency. Ferraro et al. (1993) introduced the "double normalized ratio", defined as:

$$
R_{\mathrm{BSS}}=\frac{\left(N_{\mathrm{BSS}} / N_{\mathrm{BSS}}^{\mathrm{tot}}\right)}{\left(L^{\text {sampled }} / L_{t o t}^{\mathrm{sampled}}\right)},
$$

where $N_{\mathrm{BSS}}$ is the number of BSSs counted in a given cluster region, $N_{\mathrm{BSS}}^{\text {tot }}$ is the total number of BSSs observed, and $L^{\text {sampled }} / L_{\text {tot }}^{\text {sampled }}$ is the fraction of light sampled in the same region, with respect to the total measured luminosity. The same ratio can be defined for any post-MS population. Theoretical arguments (Renzini \& Fusi Pecci 1988) demonstrate that the double normalized ratio is equal to unity for any population (such as red giant branch and horizontal branch stars, RGB and HB respectively) whose radial distribution follows that of the cluster luminosity. Ferraro et al. (2012) presented a comparison of the BSS radial distribution of 21 GCs with very different structural properties 


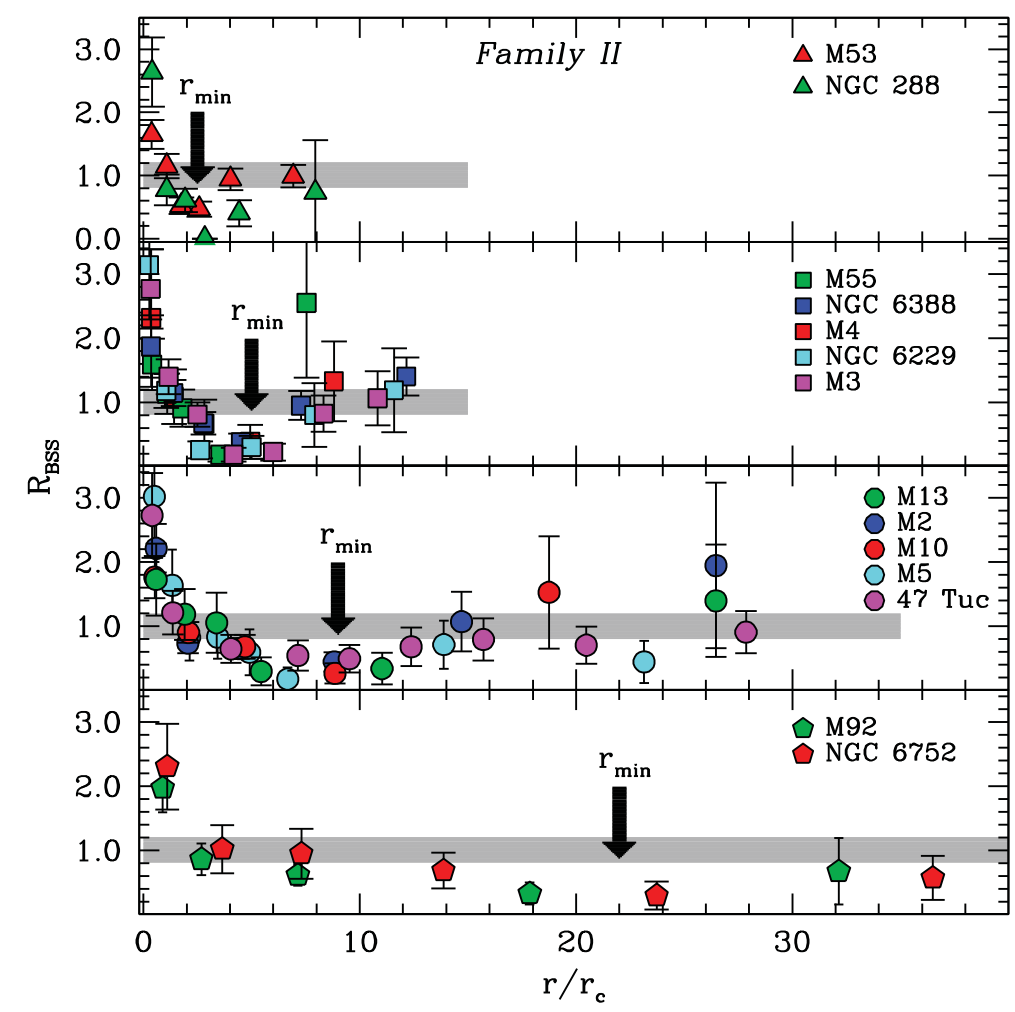

Figure 3. BSS radial distribution observed in clusters of intermediate dynamical age (Family $I I)$. The distribution is clearly bimodal and the radial position of the minimum (marked with the arrow and labelled as $r_{\mathrm{m} \text { in }}$ ) clearly moves outward from top to bottom, suggesting that the bottom clusters are more dynamically evolved than the upper ones. For the sake of clarity, the grey bands schematically mark the distribution of the reference populations. From Ferraro et al. (2012).

(hence possibly at different stages of their dynamical evolution), but with nearly the same chronological age (12-13 Gyr; Marín-Franch et al. 2009), with the only exception of Palomar 14 which formed $\sim 10.5$ Gyr ago Dotter et al. (2008). Ferraro et al. (2012) showed that once the radial distance from the centre is expressed in units of the core radius (thus to allow a meaningful comparison among the clusters), GCs can be efficiently grouped on the basis of the shape of their BSS radial distribution, and at least three families can be defined:

- Family $I$ - the radial distribution of the BSS double normalized ratio $\left(R_{\mathrm{BSS}}\right)$ is fully consistent with that of the reference population $\left(R_{\text {pop }}\right)$ over the entire cluster extension (see Figure 2);

- Family $I I$ - the distribution of $R_{\mathrm{BSS}}$ is incompatible with that of $R_{\mathrm{pop}}$, showing a significant bimodality, with a central peak and an external upturn. At intermediate radii a minimum is evident and its position $\left(r_{\min }\right)$ can be clearly defined for each sub-group (see Figure 3);

- Family $I I I$ - the radial distribution of $R_{\mathrm{BSS}}$ is still incompatible with that of the reference population, showing a well defined central peak with no external upturn (see Figure 4).

Previous preliminary analysis (Mapelli et al. 2004, Mapelli et al. 2006), Lanzoni et al. 2007a) of a few clusters indicated that BSSs generated by stellar collisions mainly/only 


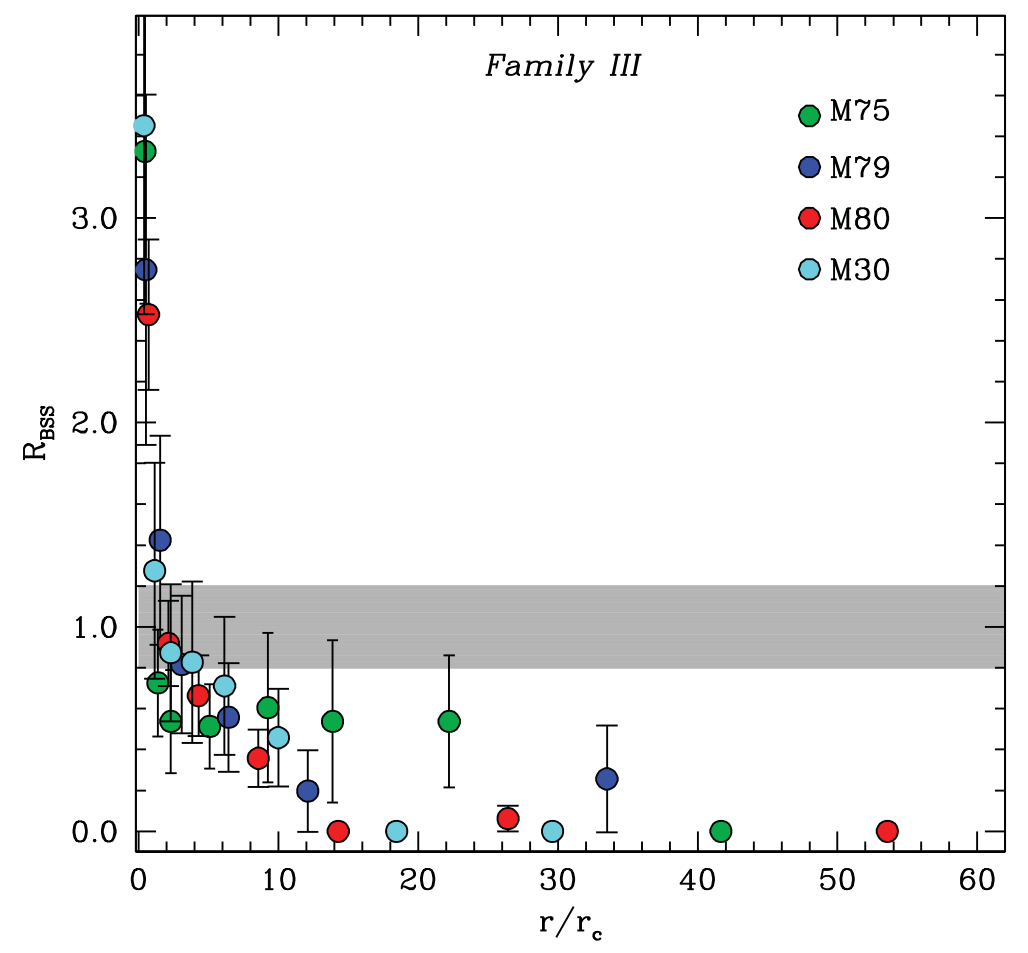

Figure 4. BSS radial distribution for dynamically old clusters (Family III): only a central peak is visible, while the external upturn is no more present because of the dynamical friction action out to the cluster outskirts. From Ferraro et al. (2012)

contribute to the central peak of the distribution, while the portion beyond the observed minimum is populated by MT-BSSs which are evolving in isolation in the cluster outskirts and have not yet suffered the effects of dynamical friction. Overall, the BSS radial distribution is primarily modelled by the long-term effect of dynamical friction acting on the cluster binary population (and its progeny) since the early stages of cluster evolution. In fact, what we call MT-BSS today is the by-product of the evolution of a $\sim 1.2 M_{\odot}$ binary that has been orbiting the cluster and suffering the effects of dynamical friction for a significant fraction of the cluster lifetime. The efficiency of dynamical friction decreases for increasing radial distance from the centre, as a function of the local velocity dispersion and mass density. Hence, dynamical friction first segregates (heavy) objects orbiting close to the centre and produces a central peak in their radial distribution. As the time goes on, the effect extends to larger and larger distances, thus yielding to a region devoid of these stars (i.e., a dip in their radial distribution) that progressively propagates outward. Simple analytical estimate of the radial position of this dip turned out to be in excellent agreement with the position of the minimum in the observed BSS radial distributions $\left(r_{\mathrm{min}}\right)$, despite a number of crude approximation (see, e.g. Mapelli et al. 2006). Moreover, a progressive outward drift of $r_{\min }$ as a function of time is confirmed by the results of direct $N$-body simulations that follow the evolution of $\sim 1.2 M_{\odot}$ objects within a reference cluster over a significant fraction of its lifetime (Miocchi et al. 2015).

In light of these considerations, the three families defined in Figs. 2-4 correspond to GCs of increasing dynamical ages. Hence, the shape of the BSS radial distribution turns out to be a powerful dynamical-age indicator. A flat BSS radial distribution (consistent 


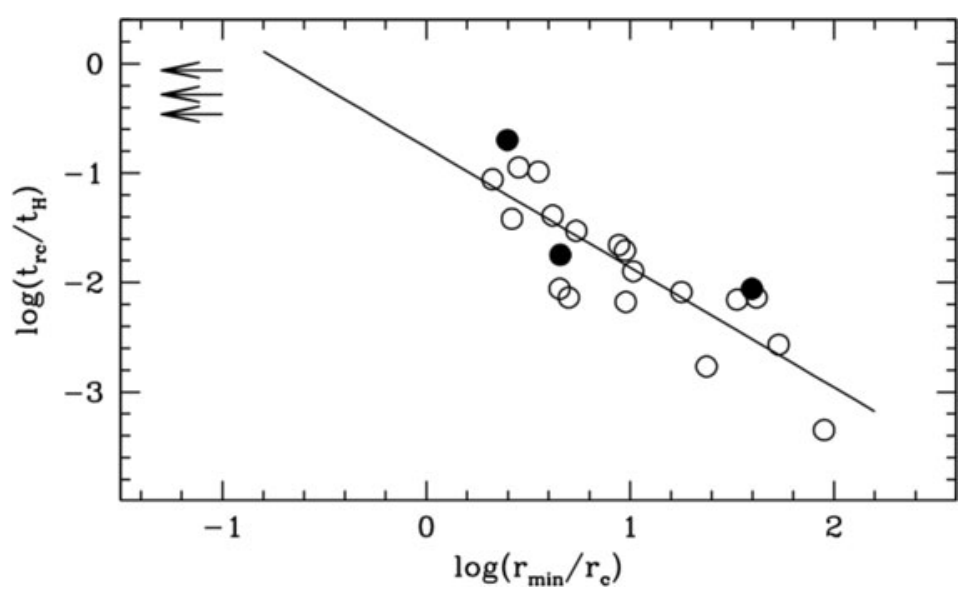

Figure 5. Core relaxation time (normalized to the Hubble time $t_{H}$ ) as a function of the time hand of the proposed dynamical clock ( $r_{\mathrm{m} \text { in }}$, in units of the core radius). Dynamically young systems (Family I) show no minimum and are plotted as lower-limit arrows at $r_{\mathrm{min}} / r_{c}=0.1$. For dynamically old clusters (Family III), the distance of the farthest radial bin where no BSSs are observed has been adopted as $r_{\mathrm{min}}$. As expected for a meaningful clock, a tight anticorrelation is found: clusters with relaxation times of the order of the age of the Universe show no signs of BSS segregation (hence their BSS radial distribution is flat and $r_{\mathrm{m} i n}$ is not definable; see Fig. 2), whereas for decreasing relaxation times the radial position of the minimum increases progressively. The solid line correspond to the best-fit relations. Empty circles are data from Ferraro et al. (2012), filled circles are three clusters (NGC362, NGC5824 and NGC5466) published after Ferraro et al. (2012).

with that of the reference population; see Family $I$ in Fig. 2) indicates that dynamical friction has not played a major role yet even in the innermost regions, and the cluster is still dynamically young. This interpretation is confirmed by the absence of statistically significant dips in the BSS distributions observed in dwarf spheroidal galaxies (Mapelli et al. 2009; Monelli et al. 2012): these are, in fact, collisionless systems where dynamical friction is expected to be highly inefficient. In more evolved clusters (Family II), dynamical friction starts to be effective and to segregate BSSs that are orbiting at distances still relatively close to the centre: as a consequence, a peak in the centre and a minimum at small radii appear in the distribution, while the most remote BSSs are not yet affected by the action of dynamical friction (this generates the rising branch of the observed bimodal BSS distributions; see upper panel in Fig. 3). Since the action of dynamical friction progressively extends to larger and larger distances from the centre, the dip of the distribution progressively moves outward (as seen in the different groups of Family II clusters; Fig. 3, panels from top to bottom). In highly evolved systems dynamical friction already affected even the most remote BSSs, which started to gradually drift toward the centre: as a consequence, the external rising branch of the radial distribution disappears (as observed for Family III clusters in Fig. 4). All GCs with a single-peak BSS distribution can therefore be classified as "dynamically old".

Interestingly, this latter class includes M30, a system that already suffered core collapse which is considered as a typical symptom of extreme dynamical evolution (Meylan \& Heggie 1997). The proposed classification is also able to shed light on a number of controversial cases debated into the literature. In fact, M4 turns out to have an intermediate dynamical age, at odds with previous studies suggesting that it might be in a PCC state (Heggie \& Giersz 2008). On the other hand, NGC 6752 turns out to be in a quite advanced state of dynamical evolution, in agreement with its observed double 


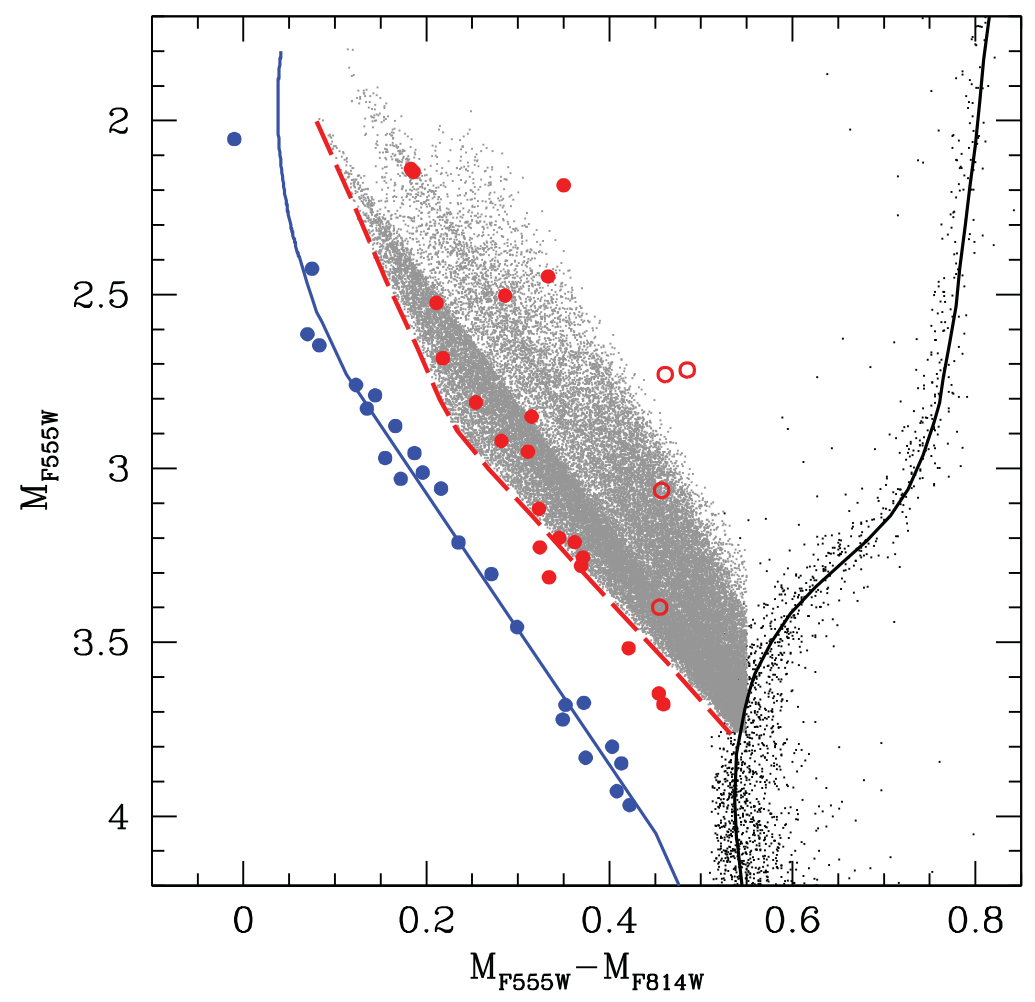

Figure 6. Distribution of the synthetic MT-binaries (grey dots) bluer than the cluster MS turnoff, in the absolute CMD of M30. The dashed line marks the "low-luminosity boundary" of the distribution. The BSSs observed by F09 along the red and the blue sequences are shown as solid circles. The positions of 4 additional red-BSSs, not considered in F09, are also shown as empty circles. Clearly, the distribution of synthetic BSSs well samples the location of the observed red-BSSs, and its low-luminosity boundary nicely follows the red-BSS sequence. The solid line is the collisional 2 Gyr-isochrone by Sills et al. (2009), which has been found to nicely fit the observed blue-BSS sequence.

King profile indicating that the cluster core is detaching from the rest of the cluster structure (Ferraro et al.2003b). Finally this approach might provide the key to discriminate between a central density cusp due to core collapse (as for M30) and that due to the presence of an exceptional concentration of dark massive objects (neutron stars and/or the still elusive intermediate-mass black holes; (see the case of NGC 6388, Lanzoni et al. 2007c).

The quantization in distinct age-families is of course an over-simplification, while the position of $r_{\min }$ is found to vary with continuity as a sort of clock time-hand. This allowed Ferraro et al. (2012) to define the first empirical clock able to measure the dynamical age of a stellar system from pure observational quantities (the "dynamical clock"): as the engine of a chronometer advances the clock hand to measure the time flow, in a similar way the progressive sedimentation of BSSs towards the cluster centre moves $r_{\text {min }}$ outward, thus marking its dynamical age. This is indeed confirmed by the tight correlations found between the clock-hand $\left(r_{\mathrm{min}}\right)$ and the central and half-mass relaxation times $\left(t_{\mathrm{rc}}\right.$ and $t_{\mathrm{rh}}$, respectively), which are commonly used to measure the cluster dynamical evolution time-scales. The correlation found with $t_{\mathrm{rc}}$ is shown in Figure 5 . Note that, while $t_{\mathrm{rc}}$ and $t_{\mathrm{rh}}$ provide an indication of the relaxation timescales at specific radial distances from the cluster centre $\left(r_{\mathrm{c}}\right.$ and $r_{\mathrm{h}}$, respectively), the dynamical clock here defined provides 
instead a measure of the global dynamical evolution of the systems, because the BSS radial distribution simultaneously probes all distances from the cluster centre.

\section{The discovery of the double BSS sequence}

While the proposed BSS formation mechanisms could be separately at work in clusters with different densities (Ferraro et al. 1995, Ferraro et al. 2006b, Lovisi et al. 2013) a few pieces of evidence are now emerging suggesting that they could also act simultaneously within the same cluster. Indeed the discovery of a double BSS sequence in M30 (Ferraro et al. 2009b, hereafter F09) indicates that this can be the case: two well distinct BSS sequences, almost parallel and similarly populated, have been found in the CMD of M30 (F09). Similar features have been detected also in NGC 362 by Dalessandro et al. (2013) and NGC 1261 by Simunovic et al. (2014).

Such a discovery has potentially opened the possibility to photometrically distinguish collisional BSSs from MT BSSs in the same cluster. In fact, the blue-BSS sequence observed in M30 is nicely reproduced by collisional isochrones (Sills et al. 2009) with ages of 1-2 Gyr. In contrast, the red-BSS population is far too red to be consistent with collisional isochrones of any age. F09 suggested that the location of the red-BSS population in the CMD correspond to the extrapolation of the the "low-luminosity boundary" outlined by the MT binary populations simulated by Tian et al. (2006) for the open cluster M67. Indeed, by using binary evolution models specifically computed for M30, Xin et al. (2015) demonstrated that the distribution of synthetic MT-BSSs in the CMD nicely matches the observed red BSS sequence (see Figure 6), thus providing strong support to the MT origin for these stars. In addition the CMD distribution of synthetic MT-BSSs never attains the observed location of the blue BSS sequence, thus reinforcing the hypothesis that the latter formed through a different channel (likely collisions).

\section{Acknowledgements}

Most of the results discussed in this talk have been obtained within the project CosmicLab (PI: Ferraro, see http:://www.cosmic-lab.eu), a 5-year project funded by the European European Research Council under the 2010 Advanced Grant call (contract ERC2010-AdG-267675). I warmly thank the other team members involved in this research: Barbara Lanzoni, Emanuele Dalessandro, Alessio Mucciarelli, Yu Xin, Licai Deng, Giacomo Beccari, Paolo Miocchi, Mario Pasquato.

\section{References}

Alessandrini, E., Lanzoni, B., Miocchi, P., Ciotti, L., \& Ferraro, F. R,. 2014, ApJ, 795, 169

Bailyn, C. 1992, ApJ, 392, 519

Bailyn, C. D., 1995, ARAA, 33, 133

Beccari, G., Sollima, A., Ferraro, F. R., et al., 2011, ApJ, 737, L3

Beccari, G., Dalessandro, E., Lanzoni, B., et al., 2013, ApJ, 776, 60

Bellazzini, M., Pasquali, A., Federici, L., Ferraro, F. R., \& Pecci, F. F. 1995, ApJ, 439, 687

Benz, W. \& Hills, J. G., 1987, ApJ, 323, 614

Buonanno, R., Corsi, C. E., Buzzoni, A., et al., 1994, A\&A, 290, 69

Chen, X. F., \& Han, Z. W., 2009, MNRAS, 395, 1822

Contreras Ramos, R., Ferraro, F. R., Dalessandro, E., Lanzoni, B., \& Rood, R. T., 2012, ApJ, 748,91

Dalessandro, E., Lanzoni, B., Ferraro, F. R., Vespe, F., Bellazzini, M., \& Rood, R. T., 2008a, ApJ, 681, 311 
Dalessandro, E., Lanzoni, B., Ferraro, F. R., Rood, R. T., Milone, A., Piotto, G., \& Valenti, E., 2008b, ApJ, 677, 1069

Dalessandro, E., Ferraro, F. R., Massari, D., Lanzoni, B., Miocchi, P., Beccari, G., Bellini, A., Sills, A., Sigurdsson, S., Mucciarelli, A., \& Lovisi, L., 2013, ApJ, 778, 135

De Marco, O., Lanz, T., Ouellette, J. A., Zurek, D., \& Shara, M. M., 2004, ApJ, 606, L151

Davies, M. B., Piotto, G., \& de Angeli, F., 2004, MNRAS, 349, 129

Dotter, A., Sarajedini, A., \& Yang, S.-C., 2008, AJ, 136, 1407

Ferraro F. R., Pecci F. F., Cacciari C., Corsi C., Buonanno R., Fahlman G. G., \& Richer H. B., 1993, AJ, 106, 2324

Ferraro, F. R., Fusi Pecci, F., \& Bellazzini, M., 1995, A\&A, 294, 80

Ferraro, F. R., et al.,Paltrinieri, B., Fusi Pecci, F., Cacciari, C., Dorman, B., Rood, R. T., Buonanno, R., Corsi, C. E., Burgarella, D., \& Laget, M., 1997, A\&A A, 324, 915

Ferraro, F. R., Paltrinieri, B., Rood, R. T., \& Dorman, B., 1999a, ApJ, 522, 983

Ferraro, F. R., Messineo, M., Fusi Pecci, F., et al., 1999b, AJ, 118, 1738

Ferraro, F. R., D’Amico, N., Possenti, A., Mignani, R. P., \& Paltrinieri, B., 2001, ApJ, 561,337

Ferraro, F. R., Sills, A., Rood, R. T., Paltrinieri, B., \& Buonanno, R., 2003a, ApJ, 588, 464

Ferraro, F. R., Possenti, A., Sabbi, E., et al., 2003b, ApJ, 595, 179

Ferraro, F. R., Beccari, G., Rood, R. T., Bellazzini, M., Sills, A., \& Sabbi, E. , 2004, ApJ, 603, 127

Ferraro, F. R., Sollima, A., Rood, R. T., Origlia, L., Pancino, E., \& Bellazzini, M., 2006a, ApJ, 638,433

Ferraro, F. R., Sabbi, E., Gratton, R., et al., 2006b, ApJ, 647, L53

Ferraro, F. R., Dalessandro, E., Mucciarelli, A., et al., 2009a, Nature, 462, 483

Ferraro, F. R., Beccari, G., Dalessandro, E., et al., 2009b, Nature, 462, 1028 (F09)

Ferraro, F. R., Lanzoni, B., Dalessandro, E., et al., 2012, Nature, 492, 393, (Ferraro et al. (2012))

Fiorentino, G., Lanzoni, B., Dalessandro, E., et al., 2014, ApJ, 783, 34

Fusi Pecci, F., Ferraro, F. R., Corsi, C. E., Cacciari, C., \& Buonanno, R., 1992, ApJ, 104, 1831

Gilliland, R. L., Bono,G., Edmonds, P. D., et al., 1998, ApJ, 507, 818

Glebbeek, E., Pols, O. R., \& Hurley, J. R., 2008, A\&GA, 488, 1007

Gosnell, N. M., Mathieu, R. D., Geller, A. M., Sills, A., Leigh, N., \& Knigge, C., 2014, ApJ, $783, \mathrm{~L} 8$

Heggie, D. C., \& Giersz, M., 2008, MNRAS, 389, 1858

Hut, P., McMillan, S., \& Romani, R. W., 1992, ApJ, 389, 527

Hills, J., \& Day, C., 1976, Astron. Lett., 17, 87

Hurley, J. R., Tout, C. A., Aarseth, S. J., \& Pols, O. R., 2001, MNRAS, 323, 630

Knigge, C., Leigh, N., \& Sills, A., 2009, Nature, 457, 288

Lanzoni, B., Dalessandro, E., Perina, S., Ferraro, F. R., Rood, R. T., \& Sollima, A., 2007a, ApJ, 670,1065

Lanzoni, B., Sanna, N., Ferraro, F. R., et al., 2007b, ApJ, 663, 1040

Lanzoni, B., Dalessandro, E., Ferraro, F. R., et al., 2007c, ApJ, 668, L139

Lanzoni, B., Ferraro, F. R., Dalessandro, E., et al., 2010, ApJ, 717, 653

Leigh, N., Sills, A., \& Knigge, C., 2007, ApJ, 661, 210

Leigh, N., Knigge, C., Sills, A., Perets, H. B., Sarajedini, A., \& Glebbeek, E., 2013, MNRAS, 428,897

Lenoard, P. J. T., 1989, AJ, 98, 217

Leonard, P. J. T., \& Linnell, A. P., 1992, AJ, 103, 1928

Lombardi, Jr., J. C., Rasio, F. A., \& Shapiro, S. L., 1995, ApJ, 445, L117

Lombardi, Jr., J. C., Warren, J. S., Rasio, F. A., Sills, A., \& Warren, A. R., 2002, ApJ, 568, 939

Lovisi, L., Mucciarelli, A., Lanzoni, B., Ferraro, F. R., Dalessandro, E., \& Monaco, L., 2013, ApJ, 772, 148

Mapelli, M., Sigurdsson, S., Colpi, M., Ferraro, F. R., Possenti, A., Rood, R. T., Sills,A., \& Beccari, G., 2004, ApJ, 605, L29

Mapelli, M., Sigurdsson,S., Ferraro, F. R., Colpi, M., Possenti, A., \& Lanzoni, B., 2006, MNRAS, 373,361

Mapelli, M., Ripamonti, E., Battaglia, G., et al., 2009, MNRAS, 396, 1771 
Miocchi, P., Pasquato, M., Lanzoni, B., et al. 2015, ApJ, 799, 44

Marín-Franch, A., Aparicio, A., Piotto, G., et al., 2009, ApJ, 694, 1498

Mathieu, R. D., \& Geller, A. M., 2009, Nature, 462, 1032

McCrea, W. H., 1964, MNRAS, 128, 147

Meylan, G. \& Heggie, D. C. 1997, ARAA, 8, 1

Monelli, M., Cassisi, S., Mapelli, M., et al., 2012, ApJ, 744, 157

Ouellette, J. A. \& Pritchet, C. J., 1998, AJ, 115, 2539

Paresce, F., de Marchi, G.,\& Ferraro, F. R., 1992, Nature, 360, 46

Pooley, D., \& Hut, P., 2006, ApJ, 646, L143

Piotto, G., et al., 2004, ApJ, 604, L109

Preston, G. W., \& Sneden, C., 2000, AJ, 120, 1014

Ransom, S. M., Hessels, J. W. T., Stairs, I. H., et al., 2005, Science, 307, 892

Renzini, A., \& Fusi Pecci, F., 1988, ARAA, 26, 199

Shara, M. M., Saffer, R. A., \& Livio, M., 1997, ApJ, 489, L59

Sills, A., Karakas, A., \& Lattanzio, J., 2009, ApJ, 692, 1411

Simunovic, M., Puzia, T. H., \& Sills, A., 2014, ApJ, 795, L10

Sollima, A., Lanzoni, B., Beccari, G., Ferraro, F. R., \& Fusi Pecci, F., 2008, A\&A A, 481, 701

Tian, B., Deng, L.-C., Han, Z.-W., \& Zhang, X.-B., 2006, A\&SA, 455, 247

Xin, Y., Ferraro, F. R., Lu, P., et al. 2015, ApJ, 801, 67 